\title{
ANÁLISE DO EFEITO DA MASSA MAGRA E DA IDADE SOBRE A TAXA METABÓLICA BASAL EM JOGADORES DE FUTEBOL
}

\section{ANALYSIS OF THE LEAN MASS AND AGE EFFECT UPON SOCCER PLAYERS' BASAL METABOLIC RATE}

\author{
Daniel Ribas da Costa ${ }^{1}$, Marcia Helena Appel ${ }^{2 *}$ \\ ${ }^{1}$ Faculdade Santana, Ponta Grossa, Paraná, Brasil, ${ }^{2}$ Universidade Estadual de Ponta \\ Grossa, Departamento de Biologia Estrutural Molecular e Genética, Paraná, Brasil \\ *Autor correspondente: Universidade Estadual de Ponta Grossa, Setor de Ciências \\ Biológicas e da Saúde, Av. Carlos Cavalcanti, 4748, Uvaranas, Ponta Grossa, Paraná, Brasil, \\ e-mail: marciaappel@yahoo.com.br
}

\section{RESUMO}

O futebol profissional exige grande vigor e dedicação aos treinamentos físicos. Assim, deslocando a composição corporal para o aumento da massa magra. O objetivo deste estudo foi analisar a composição corporal, a idade e a taxa metabólica basal (TMB) de jogadores de futebol quanto sua posição de jogo. Os voluntários da pesquisa foram 25 atletas profissionais de futebol do sexo masculino da série $\mathrm{D}$ do campeonato brasileiro de 2011. A composição corporal foi avaliada através da impedância bioelétrica, seguindo o protocolo da American College of Sports Medicine(ACSM). A análise dos dados foi realizada por meio de ANOVA (pós-teste t). A idade média dos atletas foi de $24 \pm 3,43$ (anos) e a TMB média de 1990,2 \pm 140 (kcal/dia). Quando os dados foram relacionados à posição de jogo, foi observado que os grupos goleiro e zagueiro apresentaram maior TMB e estatura que os grupos de lateral, meio e atacante. Quando a TMB foi relacionada à idade, notou-se que com o aumento da idade a TMB diminui, porém observou-se variações individuais. A TMB varia para o grupo estudado segundo a posição de jogo e a idade, porém fatores individuais foram importantes para compor a TMB.

Palavras-chave: posição de jogo, gasto calórico, composição corporal

\begin{abstract}
Professional soccer demands great physical effort and dedication during the training sessions. So, shifting body composition towards lean mass augment. The aim of the study was to analyze body composition, age and basal metabolic rate (BMR) of professional soccer players toward field position. The subjects of the study were 25 professional soccer players of Brazilian Soccer Championship D-league. Body mass composition was evaluated using bioelectrical impedance analysis following American College of Sports Medicine (ACSM) protocol. Descriptive statistic analysis was applied to data. Those were presented as mean and standard deviation. Athletes' average age was $24 \pm 3,43$ (years) and TBM average was 1990,2 \pm 140 ( $\mathrm{kcal} /$ day). Relating BMR to the field position it was observed that goalkeeper and back players had higher BMR and stature compared to side midfielders, central midfielder and forward players. Despite, some individual variations were observed. Relating TMB to age, it was noticed that TMB decayed, as age increased.
\end{abstract}

Keywords: field position, caloric spend, body composition 


\section{INTRODUÇÃO}

O futebol que veio ao Brasil por Charles Muller, um paulistano nascido no bairro do Brás, após passar um tempo na Inglaterra, volta ao Brasil trazendo uma modalidade crescente em toda Europa que se encaixava com os padrões da população daquela época.

Sendo praticado somente pela elite, mas com o passar dos tempos se tornou um esporte popular de fácil acesso para praticantes de diversas idades e de várias classes sociais. Essa é uma realidade no mundo todo, onde o futebol se tornou parte da vida da população como um todo e não apenas dos atletas. Devido a grande imagem do futebol no planeta, cada vez mais cresce o número de atletas dessa modalidade (JUNGE et al., 2002).

"A transformação do esporte mais popular do planeta Terra em "vitrine constante" e, é verdade, em algo globalizante (no sentido de amplitude e de acesso rápido) vem fazendo que os garotos e/ou pré-adolescentes "entendam" o futebol como uma atividade naturalmente fácil de ser exercida, e ainda mais fácil de ser alcançada." (ALCÂNTARA, 2006).

O futebol globalizado tem influenciado cada vez mais a mente dos jovens e futuros atletas, que vêm na modalidade ícones e ídolos os quais sonham ser um dia (Klein, 2010).

Com o inicio das competições, iniciou-se as rivalidades entre equipes, que começaram a buscar sempre melhores resultados, essas equipes são tanto clubes como nações.

"Há várias copas do mundo, das várias modalidades. Todos os anos são realizados diversas copas do mundo, ou simplesmente mundiais, de algum esporte... Copa do mundo disso ou daquilo. Futebol, porém, é o único caso que não precisa explicar: basta dizer Copa do Mundo e, portanto, sabe-se que o tema é futebol." (KLEIN, 2010).

Para alcançar os melhores resultados, foi necessário a intervenção dos profissionais de Educação Física, com periodizações e treinamentos especializados para cada atleta em cada posição de jogo, elevando o esporte a um maior nível técnico, tático e físico.

Cabendo ao profissional da área de Educação Física selecionar os atletas com melhores qualidades físicas e desempenho para competirem em alto nível esportivo. Destaca-se que o futebol requer dos seus praticantes um alto condicionamento tanto físico quanto psicológico, a fim de alcançar o nível desejado. Para que isso ocorra, os atletas devem, além de manter um nível técnico elevado, possuir um bom condicionamento físico, tanto à nível músculo-esquelético quanto metabólico. Além das qualidades físicas como a potência muscular (para bom desempenho nos chutes e no jogo aéreo decorrentes por exemplo de cobranças de escanteio), velocidade (principalmente para os jogadores do ataque que devem ser rápidos para roubar e manter a posse de bola) e agilidade (principalmente em jogadores de defesa que devem ser rápidos em marcar e desmarcar-se).

Dentro desse entendimento das qualidades de físicas se propôs treinamentos individualizados para diversas posições, sendo elas goleiros, zagueiros (volantes), laterais (alas), meio e atacantes:

Os goleiros são atletas, geralmente, de maior estatura e massa corporal se comparado aos outros jogadores, sendo necessário treinamento de flexibilidade, potência tanto de membros inferiores como de membros superiores (por serem responsáveis pela reposição da bola), e agilidade para poder buscar a bola a qualquer momento (tempo de reação)(SUTTON et al., 2009).

O treinamento de zagueiros compreende especialmente exercícios que aumentam a agilidade e potência, sendo que esses atletas devem possuir uma grande capacidade de marcar o adversário, além de uma boa reação devido a serem em sua maioria atletas de grande estatura e massa corporal. São responsáveis por impedir os avanços de jogadores adversários e evitar as jogadas aéreas próximas do gol. Os laterais são atletas que treinam principalmente velocidade, força e resistência anaeróbia devido às distâncias que estes jogadores percorrem em um curto período de tempo, além de serem responsáveis pelos cruzamentos. Os jogadores de meio de campo submetem-se a treinamentos de potência, força e agilidade, pois estes atletas devem ser habilidosos para realizar passes tanto em curtas distâncias como longas distâncias (lançamentos), pois são responsáveis pelas cobranças de faltas, inversões de jogo, controle do ritmo de jogo, achar espaços na defesa adversária, devem possuir boa técnica nos chutes, além de boa habilidade em se desmarcar, encontrar oportunidades de passes de bola para melhor finalização, e melhor posicionamento de acordo com a situação de jogo. Os atacantes normalmente dedicam-se a treinamento de força, potência e finalização, pois estes indivíduos são responsáveis pelo chute ao gol, devem saber fintar o adversário e ter uma boa visão 
de jogo (MIRANDA et al., 2013; MORGANS et al., 2014; SUTTON et al., 2009; WITTICH et al., 1998).

Florenzano (2010) fala que o futebol é um fato social, por expressar a vida coletiva:

"O futebol pode ser apreendido enquanto fato social total seja por condensar e expressar os múltiplos aspectos da vida coletiva seja por mobilizar a totalidade do indivíduo na experiência de um jogo que se reveste continuamente de novos significados." (FLORENZANO, 2010).

Para se estudar a taxa de metabolismo basal, é necessário levar em consideração que a população de amostra é formada por atletas profissionais, facilitando a análise dos resultados. Para analisar a TMB é preciso estudar as variáveis que possivelmente interferem em seu resultado, sendo assim deve-se coletar também os dados de massa magra (massa isenta de gordura), altura, e idade, além de ser necessário seguir um protocolo específico para não alterar o resultado.

A TMB é a quantidade de energia necessária para a manutenção das funções vitais do organismo, sendo medida em condições padrão de jejum, repouso físico e mental em ambiente tranquilo com controle de temperatura, iluminação e sem ruído (BURSZTEIN et al., 1989; GARROW, 1974).

Desde o século XIX, a medição da TMB é feita através da determinação da quantidade de calor produzida pelo organismo (calorimetria direta) ou pelo cálculo de calor indiretamente (calorimetria indireta) a partir do consumo de oxigênio (VO2) e excreção de gás carbônico (VCO2) tanto para fins diagnósticos quanto nutricionais. Entretanto, somente a partir do estudo de Harris e Benedictem 1919, é que houve uma tentativa de sistematização das informações existentes sobre o metabolismo basal com o desenvolvimento de equações de predição da TMB, a partir de medidas antropométricas, já que a calorimetria não era muito disponível (ROZA; SHIZGAL, 1984).

Com a mudança de orientação na estimativa das necessidades energéticas humanas, da ingestão para o gasto energético sugerido pela Food and Agriculture Organization/World Health Organization/United Nations University houve a necessidade de atualização das informações existentes sobre o metabolismo basal, com revisão das equações de predição da TMB (SCHOFIELD, 1985). A partir de então, vários estudos têm demonstrado que as equações tendem, em sua maioria, a superestimar a TMB em várias populações, principalmente, as que vivem nos trópicos (HENRY; REES, 1991).

Sabendo que a antropometria é fundamental para qualquer esporte, inclusive o futebol, neste trabalho foi realizado um estudo transversal em jogadores de futebol para analisar o comportamento da taxa metabólica basal de repouso em relação à idade dos jogadores.

\section{MATERIAL E MÉTODOS}

\section{Casuística}

O estudo se caracteriza como pesquisa de campo transversal de análise descritiva em um time de futebol profissional da série D do campeonato brasileiro 2011.

\section{População e Amostra}

Participaram do estudo 25 jogadores de futebol profissional maiores de 18 anos, do sexo masculino, da série D do campeonato brasileiro 2011 em período pré- temporada. Os dados foram agrupados e organizados segundo o posicionamento de jogo dos atletas. Todos os participante leram e assinaram o termo de consentimento livre e esclarecido. A duração e a intensidade dos treinamentos foram determinados pelos preparadores físicos dos jogadores sem interferência do pesquisador.

\section{Medida da estatura}

A estatura foi avaliada com o atleta em posição ortostática, pés descalços e unidos, procurando pôr em contato com o instrumento de medida as superfícies posteriores do calcanhar, cintura pélvica, cintura escapular e região occipital. A cabeça orientada no plano de Frankfurt. $\mathrm{O}$ avaliador ficou em pé, ao lado direito do avaliado, a altura foi mensura com uma fita métrica fibra cirúrgica de 2 metros.

(GUEDES; GUEDES, 2006).

\section{Massa corporal}

A massa corporal foi medida com o indivíduo sem sapatos e o mínimo possível de roupas, o indivíduo manteve-se em pé, de frente para o avaliador com o olhar no horizonte e a coluna ereta. A massa corporal foi mensurada em quilogramas (KAMINSKY; WHALEY, 2003). 


\section{Impedância Bioelétrica e TMB}

As avaliações foram conduzidas colocando-se 2 eletrodos, um na mão e o outro no pé do voluntário, foram realizadas no período da tarde, os avaliados seguiram as orientações da ACSM que seguem: não comer nem beber no transcorrer de 4 horas antes do teste; não exercitar-se por 12 horas antes do teste; urinar completamente dentro de 30 minutos antes do teste; não consumir de álcool nas 48 horas que precedem o teste; não consumir nenhum diurético nos 7 dias antes do teste (a menos que seja prescrito por um médico, não sendo considerado um critério de exclusão para participar da pesquisa) (DOLEZAL; POTTEIGER, 1998; SERT; ALTINDAG; SIRMATEL, 2009).

A BIA foi utilizada para estimar as variáveis: percentagem de massa magra (músculos, fluidos corporais, vísceras e outros componentes isentos de gordura), percentagem de massa gorda, taxa metabólica basal.

\section{Instrumentos utilizados}

Foram utilizados os respectivos instrumentos: balança digital Tanita (100g); estadiômetro Cardiomed $(1 \mathrm{~mm})$ e analisador (BF-900) tetrapolar de impedância bioelétrica (BIA).

\section{Análise Estatística}

Realizou-se uma análise ANOVA (pós-teste Tukey). Para análise dos dados foi utilizado o programa SPSS versão 15.0 com significância de 5\%.

\section{RESULTADOS}

Os atletas analisados apresentaram idade média de 24 $\pm 3,43$ (anos) e taxa metabólica basal (TMB) média de 1990,2 140 (kcal/dia), sendo observável que os grupos goleiro e zagueiro apresentaram maior TMB que os grupos de lateral meio e atacante.

\section{Em relação à posição de jogo}

Tabela 1: Variáveis antropométricas em relação á posição de jogo.

\begin{tabular}{c|c|c|c|c|c}
\hline & GOLEIRO & ZAGUEIRO & LATERAL & MEIO & ATACANTE \\
\hline TMB & $2156 \pm 119$ & $2051 \pm 109$ & $1921 \pm 42,1$ & $1900 \pm 128$ & $2009 \pm 120$ \\
\hline G\% & $12,05 \pm 0,96$ & $12 \pm 1,63$ & $9,5 \pm 3,67$ & $11,9 \pm 1,74$ & $11,9 \pm 0,86$ \\
\hline MM $\%$ & $88,27 \pm 0,96$ & $88,08 \pm 1,63$ & $90,92 \pm 3,67$ & $87,81 \pm 1,74$ & $87,88 \pm 0,86$ \\
\hline ESTATURA & $184 \pm 7,53$ & $186 \pm 3,11$ & $169 \pm 4,35$ & $177 \pm 5,45$ & $177 \pm 6,14$ \\
\hline IDADE & $23 \pm 2,24$ & $26 \pm 3,60$ & $23 \pm 2,96$ & $24 \pm 3,87$ & $25 \pm 4,16$ \\
\hline
\end{tabular}

TMB (Taxa Metabólica Basal), G\% (percentual de gordura), MM\% (percentual de massa magra).

Na comparação da TMB mensurada nas diferentes posições de jogo, a TMB dos jogadores na posição goleiro foi significantemente diferente se comparada ao lateral e o jogador de meio. Já as comparações das demais posições não tiveram diferença estatística (figura 1A).

Outro parâmetro antropométrico que foi estatisticamente relevante foi a estatura mostrando que os jogadores da posição lateral tinham estatura menor que os da posição goleiro e zagueiro (figura 1D). O percentual de gordura (figura 1B0, o percentual da massa magra (figura 1C) e a idade (figura 1E) não apresentaram diferenças estatísticas significantes.

\section{Em relação à idade}

Quando a TMB foi comparada com a idade observou-se que não houve uma diminuição da TMB com o aumento da idade. O grupo goleiro apresentou menor média de idade com $23 \pm 2,24$ anos e maior TMB com $2156 \pm 119 \mathrm{kcal} /$ dia, entretanto, o grupo zagueiro apresentou maior média de idade com $26 \pm 3,60$ anos e TMB $2051 \pm 109 \mathrm{kcal} /$ dia, porém quando observado os escores de lateral, meio e atacante pode se verificar tendência da diminuição da TMB com o aumento da idade, contudo estes dados foram irregulares, onde o grupo atacante com média de idade $25 \pm 4,16$ anos e TMB $2009 \pm 120 \mathrm{kcal} /$ dia apresentou maior TMB e menor média de idade do que o grupo meio com média de idade $23 \pm 2,96$ anos e TMB $1900 \pm 128 \mathrm{kcal} /$ dia. 
Figura 1: (A) representa a TMB (kcal/g/dia). (B) representa o percentual de gordura. (C) representa o percentual da massa magra. (D) estatura (cm). (E) idade (anos). Todos os dados estão representando a média e o desvio padrão das medidas em relação a posição de jogo. Análise estatística ANOVA seguido de pós-teste Tukey para múltiplas comparações. * representa $\mathrm{p}<0,05 . \mathrm{n} \geq 5$ para cada um dos grupos.
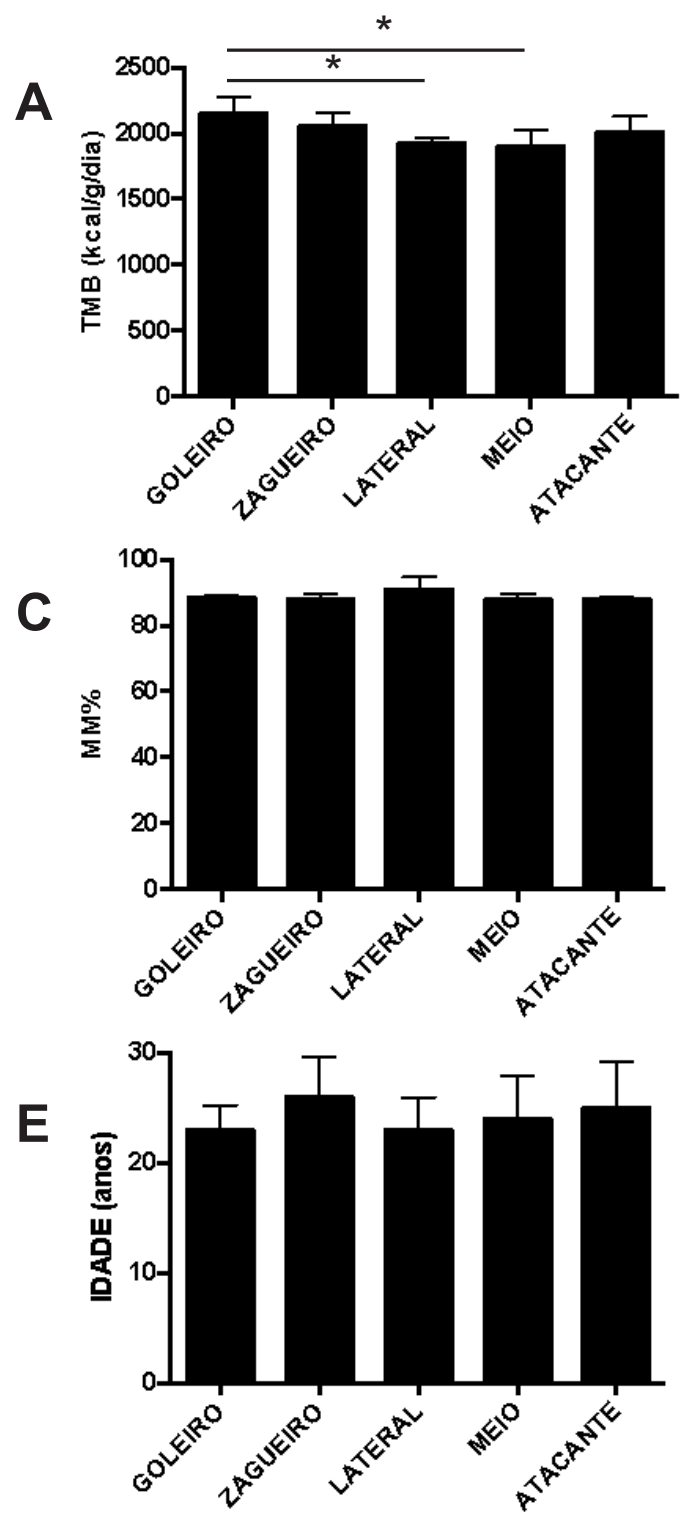
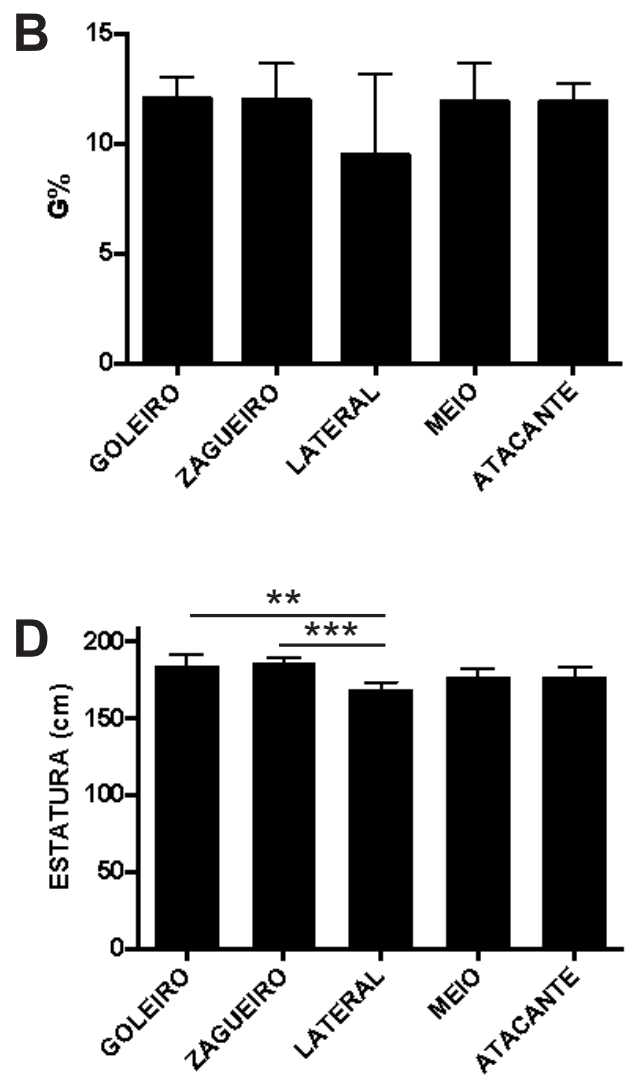

Assim, não foi possível estabelecer dentro desta faixa etária que a taxa metabólica basal diminui com a idade.

\section{DISCUSSÃO}

A análise de impedância bioelétrica (BIA) é um método rápido, não invasivo e relativamente barato para estimar a gordura corporal, apesar da exatidão da previsão relativa ser semelhante aquela do método das dobras cutâneas, a BIA pode ser preferível em algumas circunstâncias, pois não requer um alto grau de habilidade técnica, e em geral é mais confortável, requer uma cooperação mínima e interfere muito menos com a privacidade (CORNIER et al., 2011).

$\mathrm{Na}$ aplicação mais comum da BIA uma corrente de excitação de baixo nível e de uma única frequência é usada para medir a impedância corporal total. Diferentemente de uma corrente de frequência mais baixa, as frequências mais altas penetram nas membranas celulares e fluem através do líquido tanto intracelular quanto extracelular. Assim sendo, a impedância corporal total com a frequência constante de $50 \mathrm{kHz}$ reflete principalmente os volumes de água (líquido 
intracelular e extracelular) e os compartimentos musculares (DEHGHAN; MERCHANT, 2008).

De acordo com a tabela 1 , pode-se verificar que a TMB ( $\mathrm{kcal} / \mathrm{g} / \mathrm{dia})$ foi maior nas posições em que há menor deslocamento dos atletas (goleiro $=2156 \mathrm{kcal} /$ dia e zagueiro $=2051 \mathrm{kcal} /$ dia $)$ e menor nas posições em que há maior deslocamento (lateral $=1920,75 \mathrm{kcal} /$ dia, meio $=1900,12 \mathrm{kcal} /$ dia e atacante $=2008,6 \mathrm{kcal} /$ dia), também é possível notar que as posições de goleiro e zagueiro possuem maior percentual de gordura corporal $12,05 \%$ e $12 \%$ respectivamente quando comparados ás demais posições.

Quando analisado o porquê da maior TMB nos grupos goleiro e zagueiro, notou-se que os indivíduos desses grupos possuem maior estatura que os demais, e o percentual de massa magra é equivalente aos demais jogadores das outra posições. $\mathrm{O}$ que em termos absolutos faz com que tenham uma massa corporal total maior. A relação entre TMB e massa magra já é discutida a tempos em diversos estudos (LAZZER et al., 2010). No início a TMB era considerada essencialmente constante para um dado padrão corporal o que era impreciso, o que levou ao desenvolvimento de equações para predizer a TMB baseada na estatura e na massa corporal (CUNNINGHAM, 1991; ROZA; SHIZGAL, 1984).

Também, foi possível observar que os grupos goleiro e zagueiro apresentaram percentual de massa magra média de $88,27 \pm 0,96 \%$ e $88,08 \pm 1,63 \%$ respectivamente. Os grupos meio e atacante apresentaram percentual de massa magra média de $87,81 \pm 1,74 \%$ e $87,88 \pm 0,86 \%$ respectivamente, sendo assim apresentaram percentual de massa magra levemente menor que goleiro e zagueiro que por sua vez possuem maior taxa metabólica basal $2156 \pm 119 \mathrm{kcal} /$ dia e $2051 \pm 109 \mathrm{kcal} /$ dia respectivamente. Quando analisado o grupo lateral, pode-se observar que este apresenta maior percentagem de massa magra média $90,92 \pm 3,67 \%$, contudo a TMB é de $1921 \pm 42,1 \mathrm{kcal} /$ dia, isso se deve ao fato de que o grupo lateral possui menor massa magra absoluta em kg em comparação aos demais grupos. Estes dados apontam uma tendência do aumento da TMB proporcional a massa magra absoluta.

Um estudo semelhante que comparou os perfis dos jogadores pré-temporada e durante a temporada mostrou que o período não foi importante para composição corporal, contudo a posição de jogo foi um fator que teve significância sendo que os goleiros e zagueiros apresentaram maiores massa corporal total, altura, percentual de gordura absoluta e massa magra e os laterais tiveram os menores valores (LIMA et al., 2013).

A TMB teve pequena variação em relação à faixa etária por serem os voluntários jovens e com variação pequena da idade entre os grupos. Contudo, com o envelhecimento é esperado que aconteça a diminuição da TMB em decorrência da diminuição da massa individual de alguns órgãos e tecidos e da diminuição da taxa metabólica de órgãos específicos que contribuem para promoção mudanças na composição corporal (STONGE; GALLAGHER, 2010).

\section{CONSIDERAÇÕES FINAIS}

Com os resultados obtidos, foi possível verificar que o percentual de massa magra não alterou significativamente a TMB, porém os grupos com maior TMB possuem maior massa magra em $\mathrm{kg}$, os atletas com maior massa magra em $\mathrm{kg}$, também eram os atletas mais altos, demonstrando uma forte relação de estatura e massa magra com a TMB. Quando a taxa metabólica foi relacionada à idade, foi possível verificar que no grupo estudado, não houve variação significativa apesar do grupo mais novo (goleiro) possuir maior TMB que o grupo de maior idade (zagueiro).

\section{REFERÊNCIAS BIBLIOGRÁFICAS}

ALCANTARA H. A Magia do Futebol, vol.20 no.57 São Paulo May/Aug. 2006;

BURSZTEIN, S. et al. A mathematical analysis of indirect calorimetry measurements in acutely ill patients. The American journal of clinical nutrition, v. 50, n. 2, p. 227 30, ago. 1989.

CORNIER, M. et al. Assessing Adiposity: A Scientific Statement From the American Heart Association. Circulation, v. 124, n. 18, p. 1996-2019, 1 nov. 2011.

CUNNINGHAM, J. Body composition a synthetic review general prediction of energy expenditure : as a determinant and a proposed. American Journal of Clinical Nutrition, v. 54, n. 6, p. 963-69, 1991.

DEHGHAN, M.; MERCHANT, A. T. Is bioelectrical impedance accurate for use in large epidemiological studies? Nutrition journal, v. 7, n. 1, p. 26, 2008.

DOLEZAL, B. A; POTTEIGER, J. A. Concurrent resistance and endurance training influence basal metabolic rate in nondieting individuals. Journal of applied physiology (Bethesda, Md. : 1985), v. 85, n. 2, p. 695-700, ago. 1998. 
GARROW, J. S. Techniques for the measurement of human body composition. The West Indian medical journal, v. 23, n. 3, p. 165-73, set. 1974.

GuEDES, D. P.; GUEDES, J. E. R. P. Manual Prático para Avaliação em Educação Física. 1ed. ed. Barueri: Manole, 2006.

HENRY, C. J.; REES, D. G. New predictive equations for the estimation of basal metabolic rate in tropical peoples. European journal of clinical nutrition, v. 45, n. 4, p. 177 85 , abr. 1991.

JUNGE, A. et al. Prevention of soccer injuries: a prospective intervention study in youth amateur players. The American journal of sports medicine, v. 30, n. 5, p. 652-659, 2002.

KAMINSKY, L. A.; WHALEY, M. H. ACCURACY OF BMI TO DETECT OBESITY IN ADULTS. Medicine \& Science in Sports \& Exercise, v. 35, n. Supplement 1, p. S166, maio 2003

LAZZER, S. et al. Relationship between basal metabolic rate, gender, age, and body composition in 8,780 white obese subjects. Obesity (Silver Spring, Md.), v. 18, n. 1, p. 71-78, 2010 .

LIMA, C. B. N. DE et al. Estado nutricional e composição corporal de jogadores de futebol profissional. Revista Brasileira de Nutrição Esportiva, v. 3, n. 18, p. 562-569, 2013.

MIRANDA, R. E. E. P. et al. Effects of 10-week soccer training program on anthropometric, psychological, technical skills and specific performance parameters in youth soccer players. Science and Sports, v. 28, n. 2, p. 81-87, 2013.

MORGANS, R. et al. Principles and practices of training for soccer. Journal of Sport and Health Science, v. 3, n. 4, p. 251-257, 2014.

ROZA, A M.; SHIZGAL, H. M. The Harris Benedict equation reevaluated: resting energy requirements and the body cell mass. The American journal of clinical nutrition, v. 40, n. 1, p. 168-82, jul. 1984.

SCHOFIELD, W. N. Predicting basal metabolic rate, new standards and review of previous work. Human nutrition. Clinical nutrition, v. 39 Suppl 1, p. 5-41, 1985.

SERT, C.; ALTINDAG, O.; SIRMATEL, F. Determination of Basal Metabolic Rate and Body Composition With Bioelectrical Impedance Method in Children With Cerebral Palsy. Journal of Child Neurology, v. 24, n. 2, p. 237-240, 1 fev. 2009.

ST-ONGE, M.-P.; GALLAGHER, D. Body composition changes with aging: The cause or the result of alterations in metabolic rate and macronutrient oxidation? Nutrition, v. 26, n. 2, p. 152-155, fev. 2010.
SUTTON, L. et al. Body composition of English Premier League soccer players: influence of playing position, international status, and ethnicity. Journal of sports sciences, v. 27, n. 10, p. 1019-26, ago. 2009

WITTICH, A. et al. Professional football (soccer) players have a markedly greater skeletal mineral content, density and size than age- and BMI-matched controls. Calcified tissue international, v. 63, n. 2, p. 112-7, ago. 1998. 